\title{
L'anglais de spécialité chez les non-spécialistes niveau DEUG
}

\section{Pascale Fade}

\section{(2) OpenEdition}

\section{Journals}

Édition électronique

URL : http://journals.openedition.org/asp/4377

DOI : 10.4000/asp.4377

ISSN : 2108-6354

Éditeur

Groupe d'étude et de recherche en anglais de spécialité

Édition imprimée

Date de publication : 1 mars 1993

Pagination : 287-300

ISSN : 1246-8185

Référence électronique

Pascale Fade, "L'anglais de spécialité chez les non-spécialistes niveau DEUG », ASp [En ligne],

1 | 1993, mis en ligne le 01 mai 2014, consulté le 19 avril 2019. URL : http://journals.openedition.org/ asp/4377 ; DOI : 10.4000/asp.4377

Ce document a été généré automatiquement le 19 avril 2019

Tous droits réservés 


\title{
L'anglais de spécialité chez les non- spécialistes niveau DEUG
}

\author{
Pascale Fade
}

1 Dans le cadre des DEUG, que ce soit le DEUG instauré en 1973, les nouveaux DEUG ou le DEUG à venir (cf. le projet de réforme du 1er cycle), une place est toujours réservée à l'enseignement des langues, quelle que soit la discipline principale. C'est-à-dire qu'il est jugé utile ou indispensable que parallèlement à la formation disciplinaire s'effectue une formation en langues. Quelle langue étudier est à la discrétion de l'étudiant mais, dans les faits, l'anglais est choisi par une majorité écrasante d'entre eux. Pour nous, enseignants chez les non-spécialistes, deux questions immédiates se posent :

- Quel est l'impact de la discipline principale sur les besoins en anglais?

- Et, dans quelle mesure le niveau de compétence des étudiants dans leur discipline principale intervient-il pour atteindre les objectifs fixés?

2 Je vais prendre l'exemple précis des DEUG non spécialistes à Nancy 2 et vous proposer les éléments de réponse que nous avons apportés à ces questions ainsi que les interrogations qui subsistent.

3 À Nancy 2, depuis 1973, existe une équipe d'enseignants intitulée «Anglais DEUG non spécialistes »: c'est-à-dire que toutes les demandes d'enseignement de l'anglais qui proviennent des diverses disciplines nous sont adressées et nous sommes là pour apporter une réponse à ces demandes. La situation a beaucoup évolué depuis 1973 (en ce qui concerne le nombre d'étudiants en particulier) et il est utile de faire un bref historique de la situation.

4 Au départ, quatre enseignants composaient l'équipe et accueillaient les étudiants de droit, sciences économiques, psychologie, sociologie et philosophie. Entre 1974 et 1985, l'effectif est passé de 350 à 600. De nouvelles demandes émanant du campus Lettres nous ont conduits alors à nous retirer de Droit et de Sciences économiques (faute de moyens pour assurer l'ensemble) et l'équipe créée à l'origine répond donc maintenant aux besoins des sections Psychologie, Sociologie, Philosophie et Culture \& Communication. Ceci représente "bon an mal an » environ 500 étudiants. Pour être exhaustif, précisons que s'est créé à la rentrée 91/92 le S.C.E.L.V. (Service commun d'enseignement des langues 
vivantes), service commun à tout Nancy 2 qui a pour mission de gérer et d'organiser l'enseignement des langues vivantes aux non-spécialistes, toutes langues confondues.

Par rapport à l'équipe de départ, deux enseignants (dont je fais partie) sont toujours là et forment en quelque sorte le «noyau dur» de l'enseignement de l'anglais aux nonspécialistes. Il va sans dire qu'au fil des années, l'organisation de l'enseignement a beaucoup évolué et que parallèlement, les expériences dans les diverses disciplines ont alimenté notre réflexion sur « l'anglais de spécialité ».

6 La première question qui s'impose est la suivante : dans le cadre du DEUG, quel qu'il soit, quelles sont les données qui nous permettent de conclure que l'enseignement de l'anglais est un enseignement d'anglais de spécialité ?

7 Si l'on se réfère aux textes officiels, ceux de 1973 prévoyaient que $5 \%$ de l'enseignement, soit 50 heures sur l'ensemble du DEUG, étaient réservés à la langue; ces 50 heures pouvant se situer en première année exclusivement, en deuxième année ou étalées sur les deux.

Lors de l'élaboration des maquettes des nouveaux DEUG, des discussions parfois âpres et difficiles nous ont permis de situer l'anglais en deuxième année. Pourquoi insister sur ces données d'ordre administratif? Quel rapport existe-t-il entre celles-ci et l'anglais de spécialité?

Intervenir en première ou en deuxième année offre des conditions d'enseignement extrêmement différentes et influe sensiblement sur les options pédagogiques : à Nancy 2, le choix de l'anglais en deuxième année s'imposait quasiment si nous voulions un enseignement efficace et « rentable ». En effet, les effectifs imposants en première année et les moyens (horaires et humains) mis à notre disposition nous dictaient ce choix. D'autre part, la définition des besoins en deuxième année s'effectuait dans un contexte plus favorable si l'on considère qu'en fin de première année, 2 étudiants sur 3 sont en situation d'échec; en d'autres termes, en première année, comment définir les besoins d'une population qui va, en fait, disparaître aux $2 / 3$ ? Pour nous, si l'enseignement de l'anglais en DEUG se veut un enseignement adapté à son public, les moyens dont nous disposons doivent donc être investis en deuxième année. J'insiste bien sur les moyens, car il n'est pas question ici d'élitisme primaire: encadrer les premières années signifie encadrer 1500 étudiants environ qui "bénéficient» de 50 heures sur 2 ans, ce saupoudrage étant effectué par 4 enseignants.

Pour reprendre ma question « quelles sont les données qui nous permettent de conclure qu'il s'agit d'anglais de spécialité ?», il est clair que le cadre institutionnel dans lequel nous exerçons n'apporte pas de réponse évidente mais que c'est à nous, enseignants chez les non-spécialistes, de définir au mieux les modalités de notre intervention si nous voulons que le module d'anglais ait un sens, que notre enseignement ne soit pas « neutre » mais en rapport avec la spécialité. Il est intéressant de noter que dans le projet actuel de réforme du premier cycle, l'obligation de langue est bien sûr toujours là, mais que dans les propositions le module de langue intervient au premier semestre de la première année, en clair avant le choix de la discipline principale. Vous avez dit « anglais de spécialité »? 


\section{Impact de la discipline principale sur les besoins en anglais}

11 Avant de pouvoir organiser, sur le plan matériel, cet enseignement qui concerne 500 étudiants et quatre ou cinq spécialités, il est bien sûr nécessaire d'analyser les besoins. La diversité des disciplines allait-elle nous conduire à des besoins différents et multiples ?

Pour des disciplines telles que psychologie, sociologie, philosophie et sciences économiques, l'analyse nous a rapidement conduits vers la compréhension écrite. À moyen terme, de l'avis même des enseignants de la spécialité, la lecture de rapports, enquêtes ou ouvrages en anglais sera fortement recommandée et les étudiants qui s'engageront dans une recherche (maîtrise ou $3^{\mathrm{e}}$ cycle) auront une bibliographie partiellement en anglais.

13 À long terme, dans les spécialités envisagées, c'est certainement encore le support écrit qui dominera, même si d'autres besoins se font jour. Dans ces conditions, trouver des documents n'est pas un problème : les besoins existent bien car il y a matière à lire.

14 Pour un DEUG tel que Culture \& Communication, l'analyse des besoins est moins évidente : à long terme, les étudiants vont se disperser vers une multitude d'activités dont nous ne connaissons pas vraiment l'éventail puisque ce DEUG est «jeune»; à moyen terme, les choix après le DEUG vont être très divers, certains s'orientant vers une licence d'Information \& Communication, Cinéma \& Audiovisuel, Lettres modernes, Sciences du Langage, d'autres se présentant à différents concours - entre autres, concours de recrutement des instituteurs ou concours d'entrée dans des écoles professionnelles (journalisme...).

15 Les contacts avec les collègues de ces disciplines ne sont pas obligatoirement fructueux, car si d'un commun accord tous nous disent «l'anglais c'est important», la question « important pour quoi faire? » n'obtient pas de réponse claire.

16 Là encore nous avons opté pour la compréhension écrite, une des raisons étant que le travail de cette aptitude peut donner des résultats tangibles même sur un nombre d'heures limité et que les compétences de lecture acquises peuvent facilement être entretenues ensuite par les étudiants eux-mêmes. L'abondance de documents leur permet un entraînement constant.

\section{Dans quelle mesure le niveau de compétence des étudiants dans leur discipline principale intervient-il pour atteindre les objectifs fixés?}

17 L'objectif d'apprentissage, la compréhension écrite, mérite quelques précisions ou commentaires. Tout d'abord, les textes que nous étudions sont tous des textes authentiques tirés de la presse anglaise ou américaine. La liste des journaux et magazines qui servent à élaborer notre corpus est longue, mais il est intéressant de citer ceux qui sont les plus fréquemment utilisés afin de donner un cadre de référence : New Scientist, The Economist, New Society devenu New Statesman and Society, Newsweek, Observer Sunday, The Guardian, International Management et, avant sa disparition, Psychology Today. Les articles choisis ont trait à la psychologie ou la sociologie d'une part, et le langage, la 
communication, les médias d'autre part. Les titres de presse cités indiquent bien qu'il s'agit de "grande presse » en opposition à " presse spécialisée » ou ouvrages spécialisés, mais dans laquelle nous exploitons les rubriques ou les dossiers qui présentent l'étiquette recherchée.

Lire n'est pas traduire et, à partir de cette évidence, il est utile de repréciser rapidement ce que nous entendons par Compréhension écrite afin de resituer ensuite la lecture dans le contexte de la spécialité. La lecture immédiate d'un texte dans sa totalité n'est pas souhaitable dans la mesure où le lecteur va s'astreindre à une compréhension de tous les mots, ce qui débouchera fatalement sur une utilisation intensive du dictionnaire et une lecture hachée.

19 Sa vision du texte se limitera au mieux à une succession de phrases et il ne sera pas à même de l'envisager en tant que tout signifiant. Avant de s'engager dans la lecture d'un texte, le lecteur doit se mettre en condition et pour cela " interroger » le texte. La lecture du titre complétée par la lecture de l'introduction (un ou deux paragraphes dans le corpus considéré) lui permettra de découvrir le thème abordé et à partir de là d'établir un certain nombre d'hypothèses. Un survol du texte, de première phrase de paragraphe en première phrase de paragraphe, lui fera découvrir la macro-structure et les principaux sous-thèmes développés. Il sera alors en mesure d'effectuer une lecture active, à la recherche d'informations pré-localisées, et de communiquer avec le texte. Il est intéressant à cet égard de rapporter l'attitude du lecteur Bernard Pivot :

Depuis toujours j'entretiens un lien très intime avec ces pages écrites. Elles me parlent, me suscitent, et je leur réponds. (Pivot 1991 : 13)

Face à un texte, l'étudiant de deuxième année de DEUG va encore rencontrer des problèmes d'ordre lexical, grammatical ou syntaxique, d'autres liés à la cohésion textuelle ou encore à la valeur communicative du texte. Nous travaillons dans toutes ces directions (que ce soit le groupe nominal, l'étude de phrases longues, le repérage des articulateurs, les anaphoriques, un lexique "haute fréquence ", etc.) à la fois à partir d'exercices que nous avons construits en utilisant des textes de spécialité ou non, et sur les textes de spécialité.

21 L'appropriation de la méthodologie de lecture (évoquée rapidement) et un travail systématique sur les difficultés qui peuvent entraver la compréhension contribuent le plus souvent à une lecture efficace et satisfaisante pour le lecteur par rapport à ses objectifs. Toutefois certains textes vont être jugés difficiles et entraîner diverses incompréhensions, et ce pour des raisons imputables à la fois au texte et au lecteur.

Les articles qui posent peu de problèmes sont ceux qui rapportent une enquête et ses résultats, qui présentent une étude de cas ou le portrait d'un personnage connu (acteur en particulier) ou qui illustrent abondamment un fait de société. Ceux-ci ont en commun d'être des textes purement informatifs, basés sur du concret ou des faits tangibles.

La lecture est motivée par l'attrait des informations elles-mêmes, le désir d'en savoir plus, et le lecteur peut limiter son engagement au seul plaisir de lire car le texte est en quelque sorte un tout autonome qui se suffit à lui-même, avec une logique et un raisonnement internes bien sûr mais, pourrait-on dire, en vase clos, car les références externes au texte sont rares ou inexistantes. Un autre point commun est aussi l'intérêt évident du lecteur : des sujets tels que l'autisme, les horoscopes et la graphologie, les gauchers, les comportements individuels lors d'un dîner, la communication dans les affaires, sont des 
thèmes qui plaisent. Sans parler d'un texte de 5 pages sur Gérard Depardieu qui a quasiment battu tous les scores de popularité !

Quelles sont, dans les autres textes, les caractéristiques qui les rendent difficiles? Le manque d'attrait peut bien sûr être un gros handicap, mais n'explique pas tout. Deux types de texte sont à prendre en compte. Premièrement, en opposition au texte informatif, le texte qui expose des notions, des concepts et qui a pour but la réflexion autour d'un thème ou le débat d'idées. Alors même que le concept ou la notion de départ est connu du lecteur et ne représente pas un handicap en soi, alors même que le texte est structuré et progresse avec logique, le lecteur perd le fil et multiplie les erreurs de compréhension. L'observation de ces textes nous montre qu'ils renferment de nombreuses phrases très longues, entrecoupées de plusieurs incises, avec un éloignement important du sujet et du verbe, et certaines phrases paraissent effectivement interminables. Voici, pour en juger, une phrase extraite d'un texte intitulé The decay of childhood:

No one who has observed delighted parents inviting their fashionably-dressed three- or four-year-olds to parade before family and neighbours, saying 'She's going to be a heart-breaker', or 'He's a proper little he-man', or even 'Isn't she sexy' - can be surprised when these games start to invade reality.

La lecture est hachée par ces digressions ou ajouts internes, et le lecteur ne sait plus où il en est. Il est intéressant de noter que c'est dans ces textes que nous, enseignants, relevons le plus « d'erreurs » d'accord sujet/verbe : un sujet pluriel va être accompagné, beaucoup plus loin, d'un verbe au singulier ou vice-versa.

Il est vrai que l'erreur n'est pas forcément imputable à l'auteur et que nous trouvons souvent des explications du genre «le sujet pluriel est considéré comme un collectif ce qui explique le « $\mathrm{s}$ » de la troisième personne du singulier du verbe ", mais malgré tout, il semble que l'auteur absorbé dans sa réflexion arrive lui aussi à s'égarer. D'autre part, l'utilisation fréquente d'anaphoriques du type «it» ou "this » pour reprendre des pans entiers de la démonstration ne facilite pas l'intégration du raisonnement. Il apparaît aussi que ces textes exigent une attention soutenue du lecteur, et ne lui laissent guère de temps morts. Aucun exemple ou illustration ne vient ralentir le rythme et permettre l'assimilation du déjà lu avant de passer à la phase suivante. Le lecteur est constamment sollicité et un phénomène de saturation s'opère qui va conduire à - sinon l'abandon - une non-réceptivité totale. Dans ces conditions, il ne lui est plus possible d'appréhender les informations et les erreurs de compréhension vont s'accumuler.

Deuxièmement, un autre type de texte qui va poser problème est le texte qui impose au lecteur de faire appel à ses connaissances personnelles, de mobiliser son savoir pour l'intégrer au texte et à partir de là tirer profit de sa lecture. En opposition à nouveau au texte purement informatif, autonome, le texte qui s'inscrit dans un continuum et suppose une pré-connaissance va soulever des difficultés, inattendues, chez le lecteur. Alors même que le sujet n'est pas un sujet neuf pour le lecteur et/ou fait partie de sa spécialité et l'a déjà étudié, il n'arrive pas à mettre en relation ses connaissances extra-textuelles avec le texte et il se trouve démuni face à de faux problèmes, pourrait-on dire. Prenons deux exemples.

Un article qui annonçait la présence d'une exposition Lego au Science Museum mettait en cause la valeur pédagogique de l'exposition et globalement la valeur créative de ce jouet. L'auteur engageait une réflexion sur ce thème et on pourrait alors considérer que ce texte présentait les difficultés des textes précédemment évoqués. Mais en fait la description de 
certaines réalisations en Lego servait de point de référence et les manipulations possibles ou impossibles avec ces Lego constituaient le point central de sa démonstration.

Il est permis de penser que bon nombre d'étudiants avaient joué avec des Lego ou connaissaient le fonctionnement de ce jeu et pourtant des réponses erronées ou farfelues aux questions posées sur le texte prouvaient qu'ils n'allaient pas chercher, n'activaient pas leurs connaissances sur le sujet

Un deuxième article intitulé "English as a computer language » évoquait les difficultés rencontrées pour apprendre aux ordinateurs à utiliser des langues naturelles. Les approches syntaxique et sémantique étaient expliquées et un dernier programme mis au point était présenté. À nouveau, des réponses surprenantes montraient que les étudiants ne mettaient pas en relation avec le texte des connaissances pourtant utilisées dans d'autres circonstances et a priori disponibles.

31 Dans ces deux cas, la langue semble faire écran et le passage du signifiant au signifié ne s'effectue pas.

\section{3. À partir de ces réflexions quelles conclusions pouvons-nous tirer?}

32 Chez l'étudiant de deuxième année de DEUG, il est possible de conclure que, dans les cas évoqués précédemment, les difficultés rencontrées en anglais en compréhension écrite ne sont pas directement liées à son niveau de compétence dans sa discipline principale. Un commentaire s'impose. Quand nous parlons de textes de spécialité, il est important de redéfinir ce terme par rapport à notre corpus : il s'agit bien de textes qui traitent d'un sujet en rapport avec la spécialité, qui présentent une information scientifique ou une étude ou une réflexion sur un thème lié à la spécialité mais à destination en fait du connaisseur ou de « l'amateur ». C'est-à-dire que les connaissances requises pour être en mesure de lire ces textes ne sont pas celles du « spécialiste » mais celles de la «personne avertie ». En deuxième année de DEUG, les étudiants avec lesquels nous travaillons s'apparentent effectivement à la personne avertie et pas encore au spécialiste.

Une première conséquence, sur le plan pédagogique, est donc de s'adapter au niveau de connaissances des étudiants et de choisir des documents en relation avec leur compétence dans leur discipline principale tant il est vrai que, comme l'écrit Philip Riley : "The perception of a text as specialised or otherwise depends, first and foremost, on the individual's state of knowledge ». Ce qui veut dire que si nous voulons éviter le handicap de l'inconnu, il faut effectivement prendre en compte le degré de connaissance de l'apprenant dans la discipline et lui présenter des textes en rapport avec son degré de spécialisation. Comme le dit Philip Riley (1989) :

The notion of "specialisation" is best understood in terms of the relationship between an individual and discourse, rather than as a matter of formal linguistic features inherent to a given text.

Un autre point important, sur le plan pédagogique, est de se poser la question suivante : doit-on aborder uniquement des textes qui plaisent et qui «marchent bien » ou doit-on aussi présenter des textes qui, nous le savons, seront jugés difficiles et seront générateurs de problèmes?

Si nous tenons compte du fait que la motivation est un facteur important dans l'apprentissage, il est bien sûr souhaitable de favoriser les premiers. Mais nous ne devons 
pas pour autant ignorer et laisser ignorer l'existence des autres. Une première raison est que l'apprenant, en dehors de la situation de cours, rencontre ou rencontrera ces textes et par conséquent la politique de l'autruche n'est pas rentable. Une deuxième raison est que notre rôle, à nous enseignants, est de fournir les outils qui permettront à l'apprenant de mieux gérer ses difficultés. Dans le cas des textes polémiques, dont le but est le débat d'idées ou la réflexion, il convient d'élaborer des exercices spécifiques qui aideront à surmonter les obstacles mis à jour par l'observation de ces textes. La saturation du lecteur étant également un facteur défavorable, nous devons être attentifs à la longueur de ces textes et ne fournir, dans un premier temps, que des textes courts pour lesquels ce phénomène interviendra peu. En ce qui concerne les textes qui font appel aux connaissances extra-textuelles, ceux-ci doivent être l'occasion d'engager une réflexion avec les étudiants sur les moyens dont ils disposent et qu'ils doivent mettre en œuvre face à un texte pour bien ou mieux le comprendre.

Il est utile de leur rappeler que la compréhension ne passe pas seulement par l'activation de connaissances lexicales, syntaxiques ou grammaticales mais aussi par l'exploitation d'indices intra-textuels et la mobilisation de leurs connaissances en général. La coopération du lecteur avec le texte est une condition indispensable et implique qu'il apporte sa propre contribution pour que la communication s'établisse. Il faut aussi donner pour recevoir.

Pour conclure, je vais reprendre le thème de ce colloque et dire : La formation en anglais de spécialité doit s'effectuer en fonction du niveau de compétence des étudiants dans leur discipline principale. S'il est vrai qu'un texte porte l'appellation "spécialisé » selon que le lecteur est novice ou non en la matière, c'est donc bien le degré de spécialisation par rapport au public concerné qui doit être pris en compte. Il serait vain d'aborder des textes qui supposent un savoir spécifique que l'apprenant ne possède pas, le lecteur n'étant pas en mesure de "négocier» (comme dans un échange en face à face) les informations manquantes. La notion de texte de spécialité évoluera avec la spécialisation de l'apprenant, et ses connaissances de plus en plus pointues lui donneront accès à des textes de plus en plus obscurs pour le non-initié. Le rôle de l'enseignant évoluera lui aussi et sera fonction de ses propres connaissances dans le domaine. Une relation de coopération s'établira entre l'enseignant et l'apprenant, le premier apportant ses connaissances linguistiques et le second ses connaissances de la spécialité pour répondre au texte. L'enseignant sera en mesure d'avancer des hypothèses quant au contenu mais le choix final incombera au spécialiste.

\section{BIBLIOGRAPHIE}

Ludmilla Delorme et Ray Cooke. 1990. «Problématique de la sélection de documents authentiques pour la lecture ». Actes de l'Atelier Langue de spécialité du XXXe congrès de la SAES.

Pascale Fade. 1979. « Propositions pour lire ». Mélanges Pédagogiques, 31-47. 
Edith Harding. 1978. «Qu'est-ce-que les langues de spécialité ont de si spécial ? ». Mélanges Pédagogiques, 69-79.

Bernard Pivot. 1991. «Comment je lis ». Le Nouvel Observateur 17-23 octobre, 13.

Philip Riley. 1989. « Keeping secrets: ESP/LSP and the sociology of knowledge ». Mélanges

Pédagogiques, 51-61.

\section{ANNEXES}

\section{Annexe. Compte rendu de la discussion}

Le débat couvre essentiellement trois points :

1. Les étudiants ne se rendent pas compte de leurs besoins ; comment les convaincre de la nécessité de la compréhension écrite?

Une co-opération avec les enseignants de la spécialité est indispensable pour qu'ils insistent, eux aussi, sur les besoins en lecture. Il n'est pas suffisant que les enseignants de langue avertissent des demandes futures de la part des enseignants de la spécialité, ceuxci doivent les préparer en donnant des renseignements précis. Une difficulté est que la préoccupation majeure des étudiants de deuxième année de DEUG est l'obtention de leur diplôme, la langue passe au second plan. Une solution proposée serait de faire " témoigner ", en début d'année, des étudiants de maîtrise afin qu'ils fassent part de leur expérience et précisent leurs besoins réels (part d'ouvrages en anglais qui compose leur bibliographie).

2. Les étudiants n'utilisent pas leurs connaissances extra-textuelles et, globalement, n'utilisent pas les techniques de lecture déployées en langue 1 ; quelle en est la raison, quelle stratégie mettre en œuvre pour y remédier?

D'une part, un manque de confiance existe chez les étudiants et, d'autre part, lire en anglais fait porter leur attention sur la langue et non pas sur la lecture. Ils n'ont pas conscience des techniques utilisées en L1 et il est donc nécessaire d'engager une réflexion avec eux sur la lecture en L1 pour ensuite favoriser le transfert à la L2. Constatation est faite que, quel que soit le domaine (lecture, prise de notes...) le transfert de la L1 à la L2 s'effectue mal. Afin d'éviter la lecture au mot à mot, faire visualiser les informations du texte à l'aide d'un diagramme est une solution proposée. Une autre solution évoquée est de faire prendre conscience aux étudiants de la présence d'un message dans les textes et pour cela les obliger à se poser la question « quel est le message ? » et à répondre avec leurs propres mots.

3. Demande de description du fascicule « Anglais non spécialistes, Compréhension écrite » évoqué par l'intervenant.

Ce fascicule a été élaboré par l'équipe d'enseignants aux non-spécialistes de Nancy 2. Il comporte :

- une partie lexicale, qui présente des listes de mots outils, faux amis... ainsi qu'une liste de vocabulaire haute fréquence établie à partir du corpus de textes utilisés.

- une partie grammaticale, qui traite de certaines difficultés spécifiques à l'écrit : groupe nominal, incises, formes verbales complexes...

- une partie méthodologique, qui présente la méthodologie de lecture suivante : lire le titre et l'introduction du texte afin de découvrir le thème du texte, lire en continu la première phrase de chacun des paragraphes afin de découvrir la structure du texte (à 
l'aide des mots d'enchaînement) et le thème de chacune des parties. Une lecture active peut ensuite s'effectuer avec la recherche des informations essentielles qui composent chaque partie et leur organisation.

- une partie Applications : Textes.

- une partie Corrigés.

\section{AUTEUR}

\section{PASCALE FADE}

Pascale Fade enseigne à l'Université Nancy 2. pascale.fade@univ-lorraine.fr 